\title{
Aortic disease in idiopathic retroperitoneal and mediastinal fibrosis
}

\author{
M. J. MITCHINSON \\ From the Department of Pathology, University of Cambridge
}

SYNOPSIS In three patients with idiopathic mediastinal or retroperitoneal fibrosis, postmortem sections of the aorta showed aortitis (in one case) or extrusions of atheromatous debris into the inflamed adventitia (two cases). It is suggested that damage to the aortic wall, followed by adventitial inflammation due to allergy to a component of the atherosclerotic plaque, is one way in which this disease may develop.

Idiopathic retroperitoneal fibrosis not infrequently coincides with idiopathic mediastinal fibrosis (Comings, Skubi, Van Eyes, and Motulsky, 1967). In both conditions there is chronic inflammation and fibrosis, predominantly of peri-aortic distribution (Mitchinson, 1970). When the two conditions have coincided the abnormal retroperitoneal tissue has usually been in continuity with the abnormal mediastinal tissue. They are therefore considered to be manifestations of one disease.

The cause is unknown; the possibility has been raised that some damage to the aortic wall might permit leakage of an allergen from an atherosclerotic plaque into the adventitia, thus giving rise to the peri-aortic inflammation (Mitchinson, 1970). Opportunities to examine the aorta in this disease are not frequent; the following three cases in which such examination was possible lend some support to this suggested cause.

\section{Case 1 (Case 6 of Mitchinson, 1970)}

A 64-year-old man presented with 18 months' history of general malaise, which began with flatulent dyspepsia; loss of weight and shortness of breath ensued. At this time he was pale and so short of breath he could hardly walk. The pulse rate was 92 , blood pressure $120 / 80 \mathrm{~mm}$ mercury, and the liver was palpable. The apex beat was in the axilla. There was some slurring of speech and loss of memory, which he said had come on suddenly a few days before.

There was no significant previous history, except for an episode of 'cystitis' two years before. The erythrocyte sedimentation rate was $81 \mathrm{~mm}$ in the first hour (Westergren). The Wassermann test was Received for publication 17 November 1971. negative. It was thought that he was in heart failure perhaps due to the anaemia, which was normocytic, but the blood urea was found to be $99 \mathrm{mg} / 100 \mathrm{ml}$. Therefore cystoscopy and retrograde pyelography were performed. The pyelogram suggested idiopathic retroperitoneal fibrosis, with narrowing of both ureters and upper ureteric and pelvic dilatation. The renal function continued to deteriorate rapidly, in spite of leaving ureteric catheters in situ. Before a relieving operation could be performed he died suddenly.

\section{NECROPSY}

The whole aorta, from origin to bifurcation, and the proximal parts of its main branches were ensheathed in pearly-white fibrous tissue up to about 1 centimetre thick. The circumflex left coronary artery ran through a mass which 'resembled a cavernous haemangioma' in the atrioventricular groove. Both ureters were dilated as far down as their mid-course, where they were involved in the peri-aortic fibrous tissue. Both kidneys were hydronephrotic, the right being also severely infected. The duodenum and first loop of jejunum were adherent posteriorly to the peri-aortic fibrosis. The common iliac arteries were surrounded by fibrosis which was adherent to the psoas and iliacus muscles behind the vessels. The heart showed left-sided hypertrophy and dilatation, and the lungs were oedematous. Other organs were not significantly abnormal.

Histological examination showed lymphocytes, plasma cells, and Russell bodies in the peri-aortic fibrous tissue, together with characteristic secondary involvement of veins and lymphatics, Sections of aorta showed intimal thickening, mainly fibrous, and dense lymphocyte, plasma cell, and Russell body infiltration of the media. The latter was patchy in the 


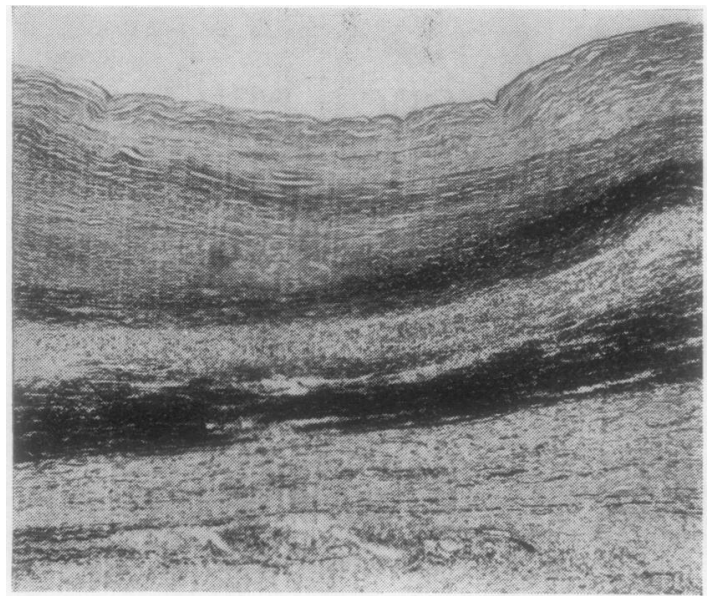

Fig. 1 Abdominal aorta (case 1), showing band-like infiltrate splitting the media, and intimal and adventitial fibrous thickening (Hart's modification of Weigert's elastic method/Ponceau $S, \times 40$ ).

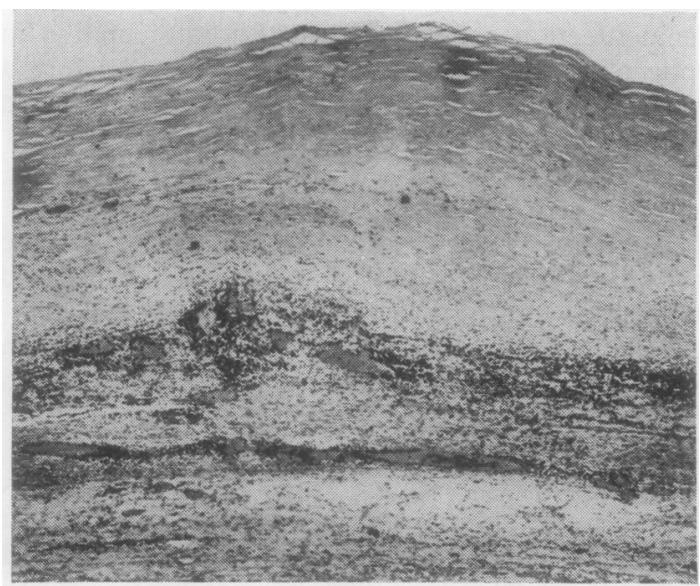

Fig. 2 Another section of the abdominal aorta (case 1) showing the density of the inflammatory infiltrate in the vascularized media (haematoxylin \& eosin, $\times 40$ ).

thoracic aorta and most severe in the abdominal aorta, where it was band-like in distribution in some sections (Figs. 1, 2). The only other histological finding of note was the mass around the left coronary artery, which proved to be only partly vascular, consisting mainly of fibrous tissue interspersed with lymphocytes, plasma cells and Russell bodies in moderate numbers.

\section{Case 2}

A 59-year-old man with myxoedema and angina pectoris complained of pain in the left groin and loss of weight. On examination the only abnormality was a blood pressure of $170 / 100 \mathrm{~mm} \mathrm{Hg}$. Intravenous pyelography showed a non-functioning left kidney and right hydronephrosis. Idiopathic retroperitoneal fibrosis was diagnosed and confirmed at operation. The ureters were dissected free from the peri-aortic plaque of fibrous tissue and left nephrostomy was performed. Three days later the patient collapsed and died.

\section{NECROPSY}

The cause of death was established as left ventricular failure with severe atherosclerotic narrowing of the coronary arteries; sections of all three arteries at various levels showed no other abnormality. The thyroid weighed $24 \mathrm{~g}$; sections showed only slight fibrosis and lymphocytic infiltration.

The retroperitoneal fibrosis was predominantly peri-aortic (Fig. 3) and around the common iliac vessels, extending upwards to within $2.5 \mathrm{~cm}$ of the left renal artery and within $5 \mathrm{~cm}$ of the right renal artery. Sections showed dense, old-looking fibrosis containing scanty foci of neutrophil leucocytes, lymphocytes, plasma cells, and Russell bodies. Atherosclerosis of the aorta was severe, and the media sufficiently disrupted at two points to allow large collections of atheromatous debris to protrude into the adventitia (Fig. 4). Inflammatory cell infiltration was present in the neighbourhood of the disrupted aortic wall and the atheromatous debris in the adventitia, but did not differ significantly in nature or amount from the foci seen elsewhere in the fibrotic adventitia.

\section{Case 3}

A 71-year-old man, who had been treated for myxoedema for 25 years, presented with breathlessness which was diagnosed and treated as a combination of left-sided heart failure and bronchitis. He os was normotensive. Clinical investigations were $N$ negative, including the Wassermann test, and a normal electrocardiogram. The ESR (Westergren) was $31 \mathrm{~mm}$ in the first hour. After repeated attacks of bronchospasm the patient suddenly collapsed and died.

NECROPSY

The left vertebral artery was atherosclerotic and occluded by recent thrombus near its termination. Both ventricles of the heart $(385 \mathrm{~g})$ were hypertrophied. The aortic valve was normal but the aortic 윽 


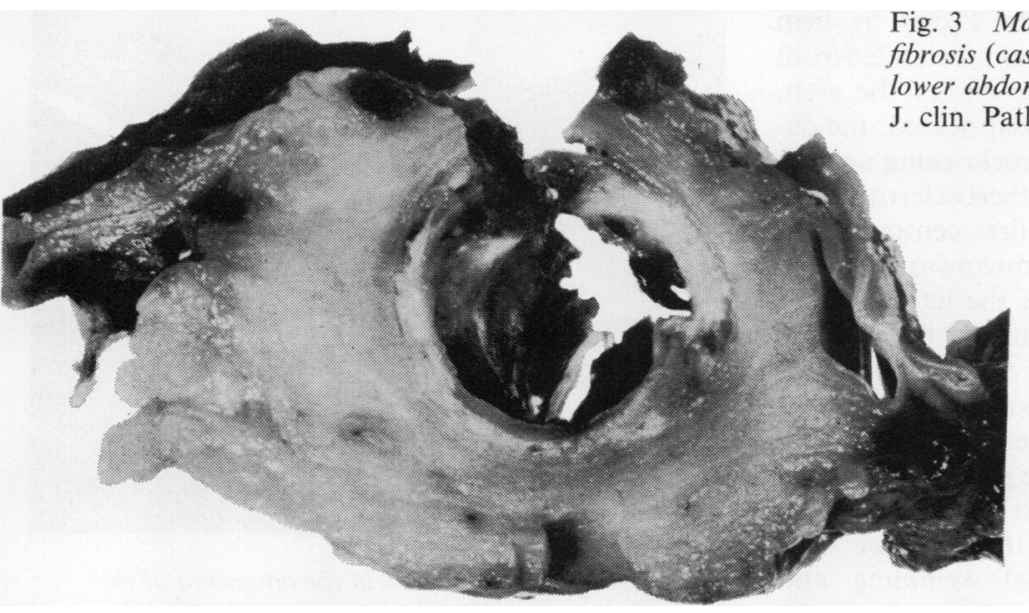

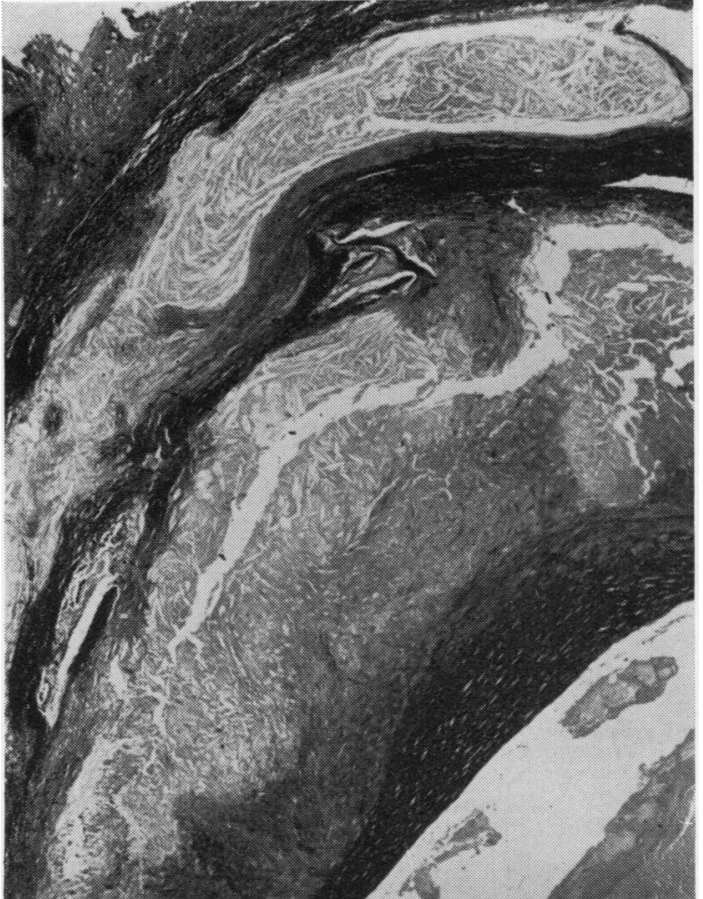

Fig. 4 Atheromatous debris in the adventitia of the abdominal aorta (case 2). The lumen is at the bottom right; the fragmented media (running from top right to bottom left) separates the intimal plaque from the adventitial atheroma (elastic PS, × 14).

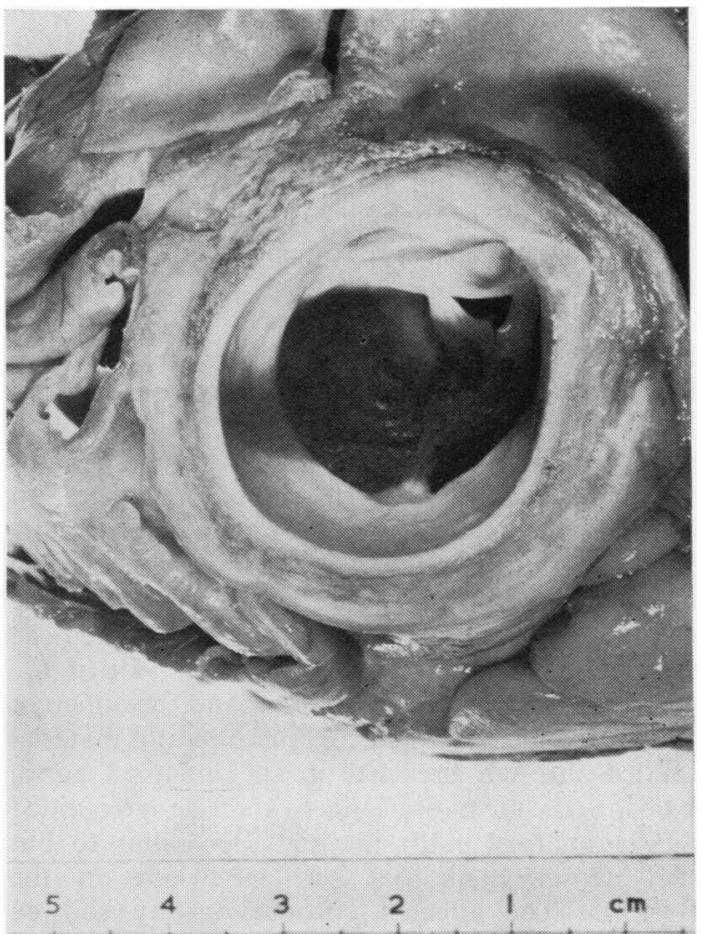

Fig. 5 Macroscopic appearance of peri-aortic fibrosis (case 3). A transverse section through the ascending aorta. 
wall was greatly thickened (up to $1 \mathrm{~cm}$ ) by firm pink-grey tissue(Fig. 5). The thickening extended from the root of the aorta as far as the end of the arch, where it gradually thinned and disappeared, the descending thoracic and abdominal aorta being normal except for a moderate degree of atherosclerosis. The thickening extended around the first centimetre of the main branches of thearch and downwardsaround the anterior descending branch of the left coronary artery for 6 centimetres. The intima of the affected part of the aorta showed scattered calcified ulcerated athererosclerotic plaques. Several structures adjacent to the thickened aorta were adherent to it, including the left lung, the bifurcation of the pulmonary artery (whose intima was covered by a thin layer of brownish thrombus), the left atrium, and the superior vena cava, which showed longitudinal wrinkling and whitish discoloration of its intima. The wall of the left inferior thyroid artery appeared to be somewhat thickened. All other arteries were unremarkable; the carotid arteries in the neck were normal except for some flecks of yellowish intimal thickening.

The thyroid gland was small, firm, pale grey and adherent to surrounding structures, especially the right lobe, which was hard and appeared to be completely replaced by fibrous tissue.

Apart from bronchopneumonia and benign prostatic hyperplasia the other organs were not remarkable.

Sections of the thoracic aorta showed dense adventitial fibrosis containing large numbers of lymphocytes and small numbers of eosinophils, plasma cells, and Russell bodies, mainly around small blood vessels. The inflammation and fibrosis involved also the outer third of the aortic media in some sections, but the inner media was usually normal. The intima showed diffuse fibrous thickening and atherosclerotic plaques. At its edges the adventitial inflammation and fibrosis infiltrated the wall of the pulmonary artery, the left side of the superior vena cava, the left atrium, and the adherent left lung. In at least two places atheromatous material extruded through breaches in the inflamed aortic media into the adventitia (Fig. 6) and was surrounded by inflammatory infiltrate (Fig. 7), similar to but much denser than that seen elsewhere in the adventitia. The affected coronary artery showed similar peri-arterial fibrosis and inflammation. In many sections the media was totally unrecognizable; the severely atherosclerotic intima was directly in contact with adventitial tissue, where it was intimately surrounded and infiltrated densely by inflammatory cells (Fig. 8). The inflammatory infiltrate around the aorta was in general less dense than around the coronary artery, except at the sites of rupture of atheromatous material into the adventitia.

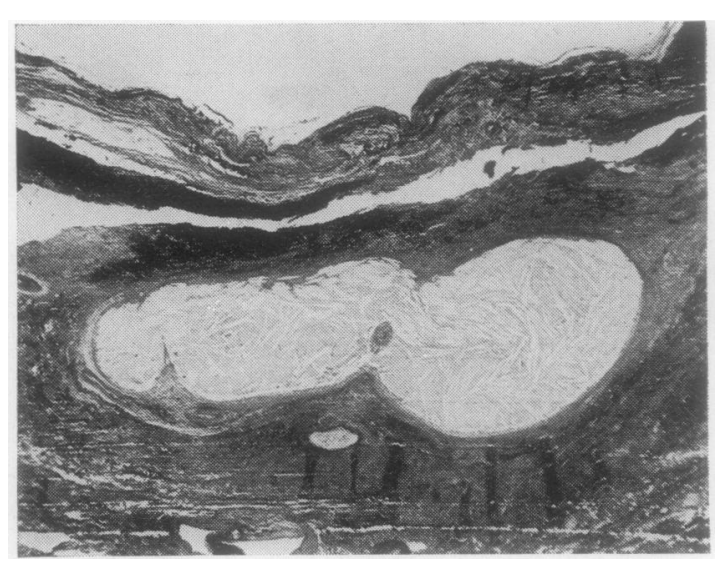

Fig. 6 Atheromatous debris in the adventitia of the thoracic aorta (case 3). The lumen is at the top; the cleft in the fragmented media is an artefact $(E P S \times 14)$.

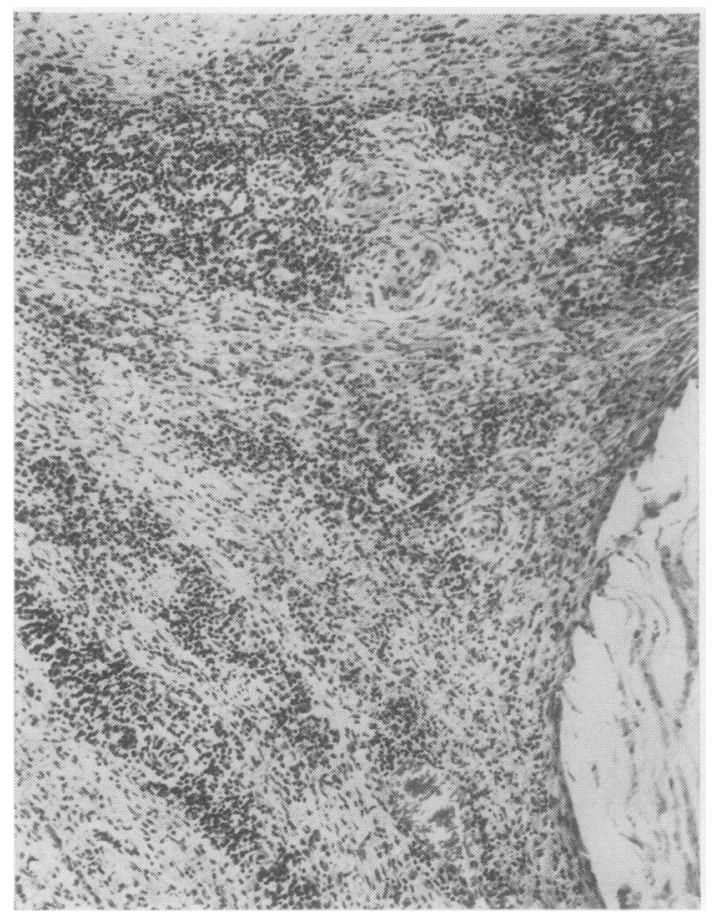

Fig. 7 Inflammatory cell infiltrate at edge of adventitial atheromatous mass (case 3$).(H \& E, \times 100)$. 


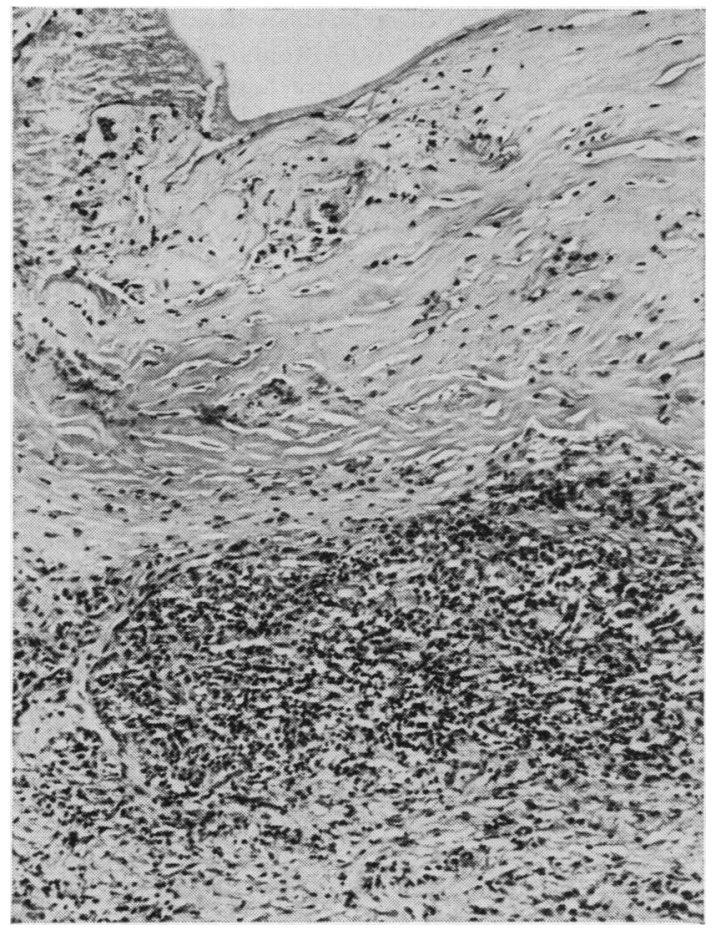

Fig. 8.

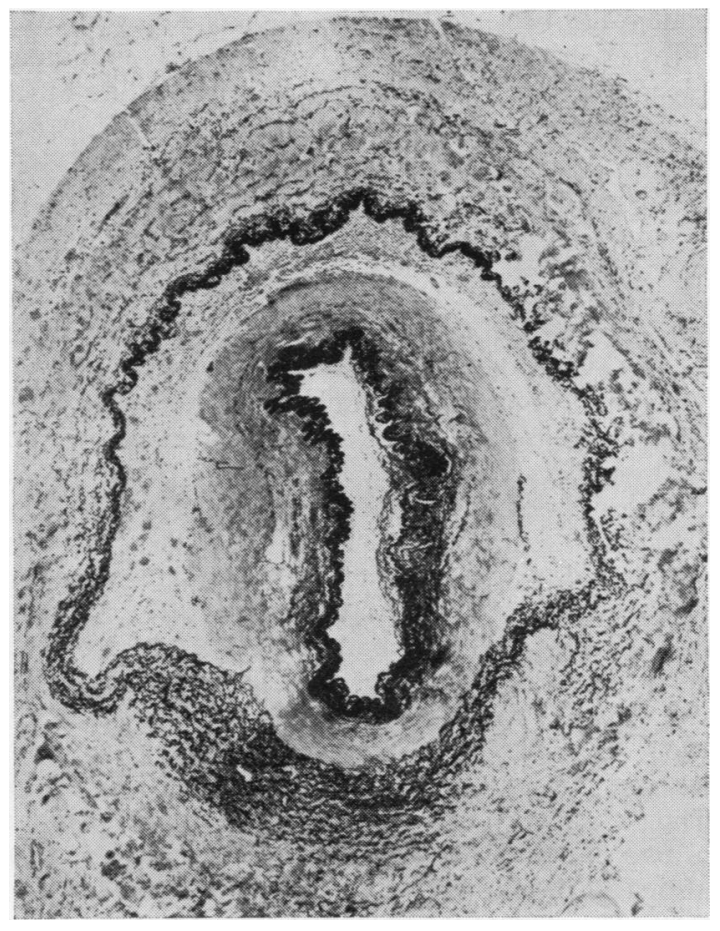

Fig. 9.

Fig. 8 Inflammatory cell infiltrate at outer edge of denuded atherosclerotic coronary intima (case 3). The lumen is at the top $(H \& E, \times 100)$.

Fig. 9 Dissecting aneurysm of left inferior thyroid artery (case 3$)(E P S, \times 40)$.

Sections of thyroid showed massive replacement by collagen containing tortuous bands of elastin, veins whose lumina had been partly or completely occluded by intimal collagenous thickening, rare remnants of thyroid acini and arteries, some of which were abnormal. The arterial abnormalities varied, some having only fibrous intimal thickening; other, larger ones showing severe disruption of internal elastic lamella and virtually complete replacement of the media by collagen. A few smaller arteries showed concentric fibro-elastic intimal thickening and partial medial replacement by collagen. Of these, only a few showed breaches in the internal elastic lamella. The rare recognizable thyroid remnants were infiltrated by lymphocytes and occasional plasma cells. In some sections the fibrous tissue had infiltrated nearby skeletal muscle.

The carotid arteries at the level of the thyroid were unremarkable, but multiple sections of the left inferior thyroid artery showed a partially healed dissecting aneurysm $(1.5 \mathrm{~cm}$ long) at the junction of media and adventitia, involving four fifths of its circumference, and associated with medial collagen accumulation (Fig. 9). The unaffected one fifth of the circumference showed multiple reduplication of the external elastic lamella. The internal elastic lamella was reduplicated, 'frayed' or lightly calcified, in various parts. The intima was slightly thickened by young-looking, mucopolysaccharide-rich connective tissue containing scanty cells, among which eosinophil leucocytes were conspicuous. The adventitia was densely fibrotic, containing recent haemorrhages, haemosiderin-laden macrophages, and a few lymphocytes and eosinophil leucocytes. The right inferior thyroid artery showed severe intimal thickening, mainly atherosclerotic, but with a few more intimal inflammatory cells than usual, including eosinophil leucocytes. The thrombosed vertebral artery showed only atherosclerosis and organizing thrombus, with no evidence of inflammation.

Sections of the (grossly normal) descending thoracic aorta showed a very scanty, probably insignificant, infiltrate of lymphocytes in adventitia and media, and slight fibrous intimal thickening of some adventitial muscular lymphatic vessels. The abdominal aorta was moderately atherosclerotic but not otherwise abnormal.

Histological examination of other organs showed 
evidence of bronchial asthma, ischaemic myocardial fibrosis, benign prostatic hyperplasia, terminal infection of the submandibular glands, and no other significant abnormality.

\section{Discussion}

The only certainty in the aetiology of idiopathic retroperitoneal fibrosis is that it can be precipitated by methysergide and alleviated by its withdrawal (Graham, Howard, LeCompte, and Sadowsky, 1966; Graham, 1967). It has usually been assumed that methysergide is acting as an allergen. However, the alternative possibility has been raised that the drug (which is a serotonin antagonist) damages the aortic wall in some way, leading to peri-aortic inflammation. The possibility is supported by observations that methysergide can apparently also produce proximal aortic damage leading to fibrosis (Graham, 1967) and probably spasm of large arteries (Ureles and Rob, 1963; Daniell, 1964; Buenger and Hunter, 1966; Rackley, Mengel, Pomerantz, and McIntosh, 1966). The occurrence of idiopathic retroperitoneal fibrosis in two patients who had taken lysergic acid (LSD), also a serotonin antagonist, has been held to lend further support to the idea (Aptekar and Mitchinson, 1970).

The histological appearances of the aortic adventitial inflammation and fibrosis 'caused' by methysergide are similar to those in the majority of patients (including the present three cases) who have not been exposed to the drug. Therefore, if the above view of methysergide's action is correct, something else must be damaging the aorta in these 'idiopathic' cases.

The three cases described here are the only ones in which the author has been able to examine several sections of the aorta, and in all of them the aorta was abnormal. In case 1 it showed severe chronic inflammation in all coats, differing only in degree of adventitial thickening from many published examples of chronic idiopathic aortitis. One other case has been described in which similar observations were made (Hardmeier and Hedinger, 1964).

If the disease resembles chronic aortitis with an unusually thick, inflamed, and fibrotic adventitia, then the severity of adventitial involvement needs to be explained. Cases 2 and 3 , especially the latter, suggest that leakage of atherosclerotic material into the adventitia may be responsible. In sections from any atherosclerotic aorta, atheromatous debris are occasionally found to have ruptured into the adventitia, but usually only a few lymphocytes and a little fibrosis are found in the neighbourhood. Such scanty adventitial inflammatory changes are also found outside atherosclerotic arteries with attenuated but $\stackrel{0}{\vec{*}}$ intact media. Schwartz and Mitchell (1962) suggested 은 that adventitial inflammation in atherosclerosis might be due to autoimmunity of some sort.

The much denser surrounding inflammatory cell reaction in case 3 certainly favours the possibility of allergy to some component of the plaque. If this interpretation is correct then it may represent a secondary phenomenon of little importance, but the is observations are equally compatible with the view $\overrightarrow{0}$ that in the cause of idiopathic retroperitoneal and mediastinal fibrosis two components are essential: $\vec{\omega}$ (1) damage to the aortic wall (by methysergide or other unknown agents) sufficient to allow excessive ? access of intimal components into the adventitia; (2) N allergy to some substance in the leaked material, $\rightarrow$ leading to chronic inflammation and thickening of N $_{\infty}$ the adventitia.

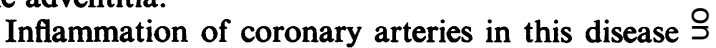
has been variously described previously by Reed and $\vec{P}$ Stinely (1959), Jones and Alexander (1966), and $\frac{7}{0}$ Russo and Esterly (1967). Case 3, and possibly case 1 , are further examples; case 3 suggests the possibility $\overrightarrow{0}$ that the coronary arteries may be affected in the N same way as the aorta.

The coincidence of thyroid disease resembling Riedel's thyroiditis in case 3 recalls similar previous associations (Hache, Woolner, and Bernatz, 1962; Raphael, Beahrs, Woolner, and Scholz, 1966; Turner-Warwick, Nabarro, and Doniach, 1966; Comings et al, 1967; Mulvaney, Gordon, and Gritti, 1968; Ullman and Dacso, 1968). It is very difficult to explain the association, although the dissecting aneurysm and possible arteritis in the inferior thyroid artery in case 3 is an unusual 용 finding. De Courcy (1942) thought that Riedel's $:$ thyroiditis was primarily an arterial disease, and 3 . this is one way in which the disease might be linked 8 to its peri-aortic counterpart. Unfortunately the present observations do not clarify the link.

It is clear that if the possible aortic origin of idiopathic mediastinal and retroperitoneal fibrosis is to be investigated further, sections of aorta at many $\underset{\sim}{\mathrm{N}}$ levels should be examined at necropsy when the $O$ opportunity presents. Immunofluorescence tech- $N$ niques might be used to investigate the role of $N$ allergy. In living patients it might be interesting to 0 search for the presence of circulating antibodies to components of the atherosclerotic plaque such as $\frac{0}{\mathbb{C}}$ lipoproteins.

I am grateful to Dr Peter Andrews, who provided the necropsy report and sections of case 1; to Drs $\overrightarrow{\mathbb{D}}$ J. C. Valentine and R. Bishop, who provided necropsy reports and fixed tissues of cases 2 and 3 respectively; 
and to Mr S. W. Patman and the Department of Medical Photography, Addenbrooke's Hospital, for the photographs.

\section{References}

Aptekar, R. G., and Mitchinson, M. J. (1970). Retroperitoneal fibrosis in two patients previously exposed to LSD. Calif. Med., 113 (2), 77-79.

Buenger, R. E., and Hunter J. A. (1966) Reversible mesenteric artery stenoses due to methysergide maleate. J. Amer. med. Ass., $198,558-560$.

Comings, D. E., Skubi, K. B., Van Eyes, J., and Motulsky, A. G. (1967). Familial multifocal fibrosclerosis. Ann. intern. Med., 66, 884-892.

Daniell, H. W. (1964). Vasospastic reaction to methysergide maleate simulating Leriche syndrome. Ann. intern. Med., 60, 881-885.

De Courcy, J. L. (1942). A new theory concerning the etiology of Riedel's struma. Surgery, 12, 754-762.

Graham, J. R. (1967). Cardiac and pulmonary fibrosis during methysergide therapy for headache. Amer. J. med. Sci., 254, 1-12.

Graham, J. R., Howard, I. S., LeCompte, P. R., and Sadowsky, N. L. (1966). Fibrotic disorders associated with methysergide therapy for headache. New Engl. J. Med., 274, 359-368.

Hache, L., Woolner, L. B., and Bernatz, P. E. (1962). Idiopathic fibrous mediastinitis. Dis. Chest, 41, 9-25.

Hardmeier, T., and Hedinger, C. (1964). Beziehungen zwischen der retroperitonäalen Fibrose und der sogenanntenTakayasuschen
Arteriitis. Schweiz. med. Wschr., 94, 1669-1676.

Jones, E. A., and Alexander, M. K. (1966). Idiopathic retroperitoneal fibrosis associated with an arteritis. Ann. rheum. Dis., 25, 356-360.

Mitchinson, M. J. (1970). The pathology of idiopathic retroperitoneal fibrosis. J. clin. Path., 23, 681-689.

Mulvaney, W. P., Gordon, L. Z., and Gritti, E. J. (1968). Another look at periureteral fibrosis. J. Urol., 99, 417-422.

Rackley, C. E., Mengel, C. E., Pomerantz, M., and McIntosh, H. D. (1966). Vascular complications with use of methysergide. Arch. intern. Med., 117, 265-269.

Raphael, H. A., Beahrs, O. H., Woolner, L. B., and Scholz, D. A. (1966). Riedel's struma associated with fibrous mediastinitis. Mayo clin. Proc., 41, 375-382.

Reed, W. G., and Stinely, R. W. (1959). Massive periaortic and periarterial fibrosis; report of a case. New Engl. J. Med., 261, 320-323.

Russo, J. V., and Esterly, J. R. (1967). Retroperitoneal and periarterial fibrosis. Arch. Path., 83, 396-398.

Schwartz, C. J., and Mitchell, J. R. A. (1962). Cellular infiltration of the human arterial adventitia associated with atheromatous plaques. Circulation, 26, 73-78.

Turner-Warwick, R., Nabarro, J. D. N., and Doniach, D. (1966). Riedel's thyroiditis and retroperitoneal fibrosis. Proc. roy. Soc. Med., 59, 596-598.

Ullmann, A. S., and Dacso, M. R. (1968). Multifocal idiopathic fibromatosis. Dis. Chest, 53, 657-659.

Ureles, A. L., and Rob, C. (1963). Acute ischemia of a limb complicating methysergide maleate therapy. J. Amer. med. Ass., 183, 1041-1042. 